function and blood glucose concentrations. The "safe" blood glucose concentration may vary according to the clinical situation, eg. during hypoxia, polycythemia, or convulsions, and may be independent of the gestational and postnatal age and birth weight. Clinical signs of hypoglycemia in the neonate are not well established and the question of neural dysfunction or damage with asymptomatic hypoglycemia is addressed in the following paper.

\title{
HYPOGLYCEMIA AND NEURAL DYSFUNCTION
}

Evoked potentials were used to measure neural function in relation to blood glucose concentration in 17 children at the Department of Child Health, University of Newcastle upon Tyne. Thirteen children were admitted for investigation of metabolic or endocrine disorders and hypoglycemia was provoked by fasting or insulin administration; 4 had recurrent episodes of spontaneous hypoglycemia. Abnormal brainstem auditory evoked potentials were recorded in 9 and abnormal somatosensory evoked potentials in 1 of the 11 children whose blood glucose concentration remained above $2.6 \mathrm{mmol} / 1 ; 5$ of these 10 children were asymptomatic and 5 became drowsy. No change in evoked potentials was recorded in the 6 children whose blood glucose concentration remained above $2.6 \mathrm{mmol} / \mathrm{l}$. Of the $10 \mathrm{children}$ with abnormal evoked potentials, 6 had normal latencies immediately after the IV administration of 258 dextrose $(2 \mathrm{ml} / \mathrm{kg})$ or the IM administration of glucagon ( $20 \mathrm{ug} / \mathrm{kg}$ ), whereas $4 \mathrm{had}$ persistently prolonged I-V latencies or absent wave $\mathrm{V}$ for $1 \mathrm{hr}$, 1.5 hrs, 16 hrs, and 2 days. The authors suggest that blood glucose concentration should be maintained above $2.6 \mathrm{mmol} / \mathrm{l}$ to ensure normal neural function in newborns and children irrespective of the presence or absence of abnormal clinical signs. (Koh THHG, Aynsley-Green A, Tarbit M, Eyre JA, Neural dysfunction during hypoglycemia. Arch Dis Child Nov 1988; 63 : 1353-1358).

COMMENT. A prospective controlled study of neonates with episodes of hypoglycemia has shown that long term follow up may reveal impairments in intellectual function (Pildes et al. Pediatrics 1974; 54:5). Short term follow up studies of infants who have suffered hypoglycemia may be misleading. Clinical signs of hypoglycemia and disturbances of neural function in the newborn baby may be subtle and difficult to recognize, and the distinction between "symptomatic" and "asymptomatic" hypoglycemia may require recordings of evoked potentials to demonstrate neural dysfunction. Whether the transient abnormalities in evoked potentials demonstrated in the above study can be predictive of permanent neural damage remains to be determined. The neurological signs resulting from abnormal cerebral metabolism secondary to hypoglycemia may depend on the rate of fall of blood sugar, the duration and degree of hypoglycemia, and the age of the patient. (Etheridge JE. 
Hypoglycemia and the central nervous system. In Symposium on Pediatric Neurology, Ed. Millichap JG, Ped Clin N Amer Nov $1967 ; 14: 865)$. Alternative substrates, particularly ketone bodies, can be used for energy by the brain and may have a protective effect during episodes of hypoglycemia. (Persson B et al. Acta Paediatr Scand 1972;61:273).

\section{HYPOGLYCEMIA AND BRAIN DAMAGE}

The neurochemical effects, patterns of cerebral blood flow derangement, time course and distribution of brain tissue damage, and selective neuronal necrosis resulting from hypoglycemia are compared to the biological effects of ischemia and epilepsy - in a neurological progress report from the Departments of Pathology and Clinical Neurosciences, University of Calgary, Alberta, Canada, and the Laboratory for Experimental Brain Research, University Hospital, Lund, Sweden. Hypoglycemia interferes with cerebral energy production, causing membrane depolarization, loss of ion homeostasis, and lipolysis with accumulation of free fatty acids. Tissue aspartate and quinolinic acid levels rise and glutamate levels in CNS tissue fall during hypoglycemia. Cerebral blood flow is increased during the insult and decreased following the insult. Energy failure is moderate in degree with ATP levels dropping to 25 to $30 \%$ of control. Unlike ischemia and epilepsy, hypoglycemia is not accompanied by lactic acidosis. The duration of hypoglycemic insult required to produce selective neuronal necrosis is 10-20 min and neuronal death occurs within 1-8 hrs. (Auer RN, Siesjo BK. Biological differences between ischemia, hypoglycemia, and epilepsy. Ann Neurol Dec 1988;24:699-707).

COMMENT. Ischemia, hypoglycemia, and epilepsy, long thought to produce similar or identical brain damage, apparently have different mechanisms and effects on brain tissue. Insults of equal duration are not equipotent in causing brain damage. Ischemia is much more potent than hypoglycemia, and hypoglycemia is more potent than status epilepticus per minute of insult. The timing of neuronal necrosis evolves over hours to days in ischemia, minutes to hours in hypoglycemia, and during or very shortly after the insult of status epilepticus.

\section{METHYLMALONIC ACIDEMIA AND EXTRAPYRAMIDAL DISEASE}

Four children, 4-13 yrs old, with methylmalonic acidemia who developed acute dystonia after metabolic decompensation with ketoacidosis are reported from the Department of Pediatrics, University of Pennsylvania School of Medicine, and the Children's Hospital of Philadelphia, PA, and other collaborating institutions. The neurological complications were the result of acute necrosis of the globus pallidus and some involvement of internal capsules. One patient, aged 5 yrs, was a poor feeder and had vomited soon after birth. She was hospitalized at 1 wk of age with lethargy and ketoacidosis, and had massive amounts of methylmalonic acid in the urine that were not reduced by IM cyanocobalamin. Treatment with long-term oral alkali, and a 